\title{
Harmonic resonances due to transmission-system cables
}

\author{
M.H.J. Bollen ${ }^{1}$, S. Mousavi-Gargari ${ }^{2}$ and S. Bahramirad ${ }^{3}$ \\ ${ }^{1}$ Electric Power Systems Group, Luleå University of Technology \\ 93187 Skellefteå, Sweden \\ math.bollen@1tu.se \\ ${ }^{2}$ Tennet, P.O.Box 718, 6800 AS Arnhem, The Netherlands \\ Shima.MousaviGargari@tennet.eu \\ ${ }^{3}$ ComEd, PO Box 805379, Chicago IL 60680-5379, USA \\ Shay.Bahramirad@ComEd.com
}

\begin{abstract}
This paper gives some examples of harmonic issues that can occur when long ac cables are connected in the transmission grid. The main impact is that resonances can occur at much lower frequencies than when only overhead lines are present. Two illustrative case studies are presented: one for a $275-\mathrm{kV}$ cable, one for a $400-\mathrm{kV}$ cable in combination with a $132-$ $\mathrm{kV}$ capacitor bank. A simple rule-of-thumb is given, to decide if a detailed harmonic study is needed. Some guidelines for such a study are given as well.
\end{abstract}

\section{Key words}

Electric power transmission, power quality, power system harmonics, high-voltage cables

\section{Introduction}

Cables introduce large capacitances to the transmission system, about 20 times as much as an overhead line of the same length. It is therefore important to consider harmonic resonances as part of the studies done before installing such cables. Resonances in the input impedance for a 150$\mathrm{kV}$ cable connecting an off-shore wind farm are studied in [1]. A resonance frequency close to $150 \mathrm{~Hz}$ is predicted from a detailed simulation. The resonance frequencies, obtained from a simple model, are presented as a function of cable length and fault level in [2]. Resonances will occur at lower frequencies than are common in transmission systems. Amplification of common harmonics like 5, 7, 11 and 13 becomes a possibility. In some cases, even amplification of the third harmonic becomes possible.

In this paper a general analysis is presented of the resonances that can occur when long ac cables are introduced in the transmission system. Section 2 of the paper will describe the distributed-parameter model needed for transmission-line cables. This model is used in Section 3 to calculate the resonance frequency and to compare the obtained frequencies with those obtained from a simple model. A number of illustrative case studies are shown in Section 4 and Section 5. The aim of these is only to illustrate the kind of resonance phenomena that may occur, not as full-scale harmonic studies. Some recommendations about harmonic studies and need for future research are given in Section 6, including a simple rule-of-thumb on when a detailed study is needed.

\section{Distributed parameter model}

A transmission-cable can be modelled in a number of ways: from a single inductance or capacitance, through a combination of a series inductance and two shunt capacitor, up to distributed series impedance and shunt capacitance. The latter model is most appropriate when studying harmonics over a wider frequency range.

The transmission cable is modelled as a "distributed transmission line". The two-port (four-pole) model for a distributed transmission line is shown in Figure 1, where it is terminated by an impedance $Z_{R}$. The model consists of one series impedance

$$
\mathrm{Z}^{\prime}=\mathrm{z} \frac{\sin \mathrm{h}(\boldsymbol{\gamma} \boldsymbol{\ell})}{\gamma \boldsymbol{\ell}}
$$

And two shunt admittances (one on each side of the line, as in a Pi-model):

$$
Y^{\prime}=Y \frac{\tanh \left(\frac{1}{2} \gamma \ell\right)}{\frac{1}{2} \gamma \ell}
$$

where $\mathbf{Z}=\mathbf{z} \boldsymbol{\ell}$ is the low-frequency series impedance, and $\mathbf{Y}=\mathbf{y} \boldsymbol{\ell}$ is the low-frequency shunt admittance, with $\boldsymbol{\ell}$ : the length of the cable.

The transfer exponent is obtained from: $\boldsymbol{\gamma}=\sqrt{\mathbf{z y}}$, with $\mathbf{z}=\mathbf{r}+\mathbf{j} \boldsymbol{\omega} \mathbf{l}$, the series impedance per unit length and $\mathbf{y}=\mathbf{j} \boldsymbol{\omega} \mathbf{c}$, shunt admittance per unit length 
The relation between receiving end $(\mathrm{R})$ and sending end (S) voltages and currents is as follows:

$$
\left[\begin{array}{l}
\mathbf{V}_{S} \\
\mathbf{I}_{S}
\end{array}\right]=\left[\begin{array}{ll}
\mathbf{A} & \mathbf{B} \\
\mathbf{C} & \mathbf{A}
\end{array}\right]\left[\begin{array}{l}
\mathbf{V}_{\mathbf{R}} \\
\mathbf{I}_{\mathbf{R}}
\end{array}\right]
$$

With the following expressions for the elements of the matrix:

$$
\begin{gathered}
A=\cosh (\gamma \ell)=\cosh (\sqrt{\mathrm{ZY}}) \\
\mathrm{B}=\mathrm{Z}_{\mathrm{C}} \sinh (\gamma \ell)=\sqrt{\frac{\mathrm{Z}}{\mathrm{Y}} \sinh (\sqrt{\mathrm{ZY}})} \\
\mathrm{C}=\frac{1}{\mathrm{Z}_{\mathrm{C}}} \sinh (\sqrt{\mathrm{ZY}})
\end{gathered}
$$

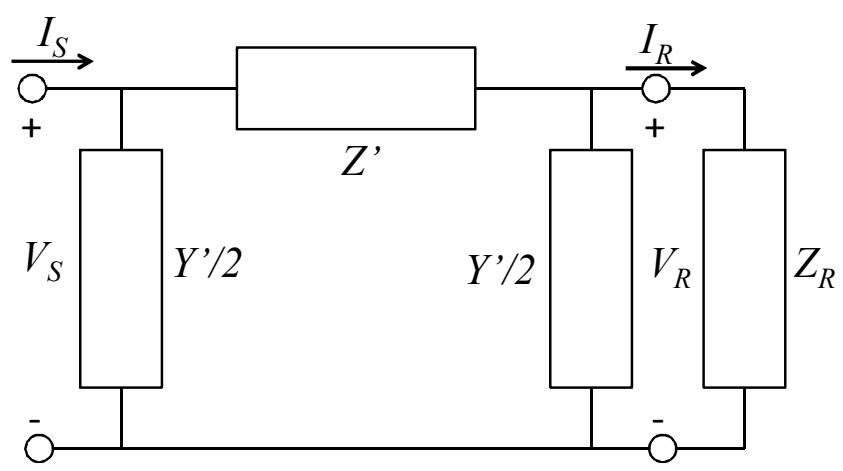

Figure 1. Cable model with termination $Z_{R}$ representing the rest of the grid.

A number of parameters as a function of frequency can be calculated from this model:

Input impedance at the sending end

$$
\frac{V_{S}}{I_{S}}=\frac{A Z_{R}+B}{C Z_{R}+A}
$$

Transfer function from sending to receiving end

$$
\frac{I_{R}}{I_{S}}=\frac{1}{C Z_{R}+A}
$$

Transfer impedance from sending to receiving end

$$
\frac{\mathbf{V}_{\mathrm{R}}}{\mathbf{I}_{\mathrm{S}}}=\frac{\mathbf{Z}_{\mathrm{R}}}{\mathbf{C Z}_{\mathrm{R}}+\mathbf{A}}
$$

\section{Resonance frequencies}

Consider a simple configuration of one cable connected to a grid with a certain source impedance. Use as a base case the following parameters:

\footnotetext{
$\checkmark \mathrm{r}=50 \mathrm{~m} \Omega / \mathrm{km}$; cable resistance per $\mathrm{km}$

$\checkmark \quad 1=0.57 \mathrm{mH} / \mathrm{km}$; cable inductance per $\mathrm{km}$

$\checkmark \quad \mathrm{c}=0.15 \mu \mathrm{F} / \mathrm{km}$; cable capacitance per $\mathrm{km}$

$\checkmark \quad \boldsymbol{\ell}=15 \mathrm{~km}$; cable length

$\checkmark$ Unom $=275 \mathrm{kV}$; rated voltage of the system
}

$\checkmark \quad \mathrm{Sk}=6000 \mathrm{MVA}$; short-circuit capacity at the receiving end

$\checkmark \quad \mathrm{XR}=1 ; \mathrm{X} / \mathrm{R}$ ratio at the receiving end

For the source impedance an $\mathrm{X} / \mathrm{R}$ ratio equal to one has been assumed. The latter may seem exceptionally small for a transmission grid. However the "source impedance" at harmonic frequencies includes damping due to resistive loads and increase of resistance with frequency.

The results from the calculations, using the distributed parameter model, have been compared with the results using a first order model of cable capacitance and source inductance only. The resonance frequencies as obtained from the distributed cable model are shown in Table 1. The resonance frequency in Table 1 is obtained as the frequency for which the transfer function had its highest value. The maxima of input impedance and transfer impedance occurred within a few Hertz from this frequency.

In Table 2, the results are shown when the resonance frequency is calculated from the commonly-known expression:

$$
f_{\text {res }}=\frac{1}{\sqrt{L C}}
$$

Where $L$ is the inductive part of the source impedance and $C$ the total cable capacitance.

Table 1. Resonance frequencies (in $\mathrm{Hz}$ ) for the combination cable - grid: distributed cable model

\begin{tabular}{|l|c|c|c|c|c|c|}
\hline \multicolumn{1}{|c}{ cable - grid: distributed cable model } \\
\hline & \multicolumn{2}{|c|}{$132 \mathrm{kV}$} & \multicolumn{2}{c|}{$275 \mathrm{kV}$} & \multicolumn{2}{c|}{$400 \mathrm{kV}$} \\
\hline & 1 & 4.1 & 6 & 15 & 9 & 32 \\
& GVA & GVA & GVA & GVA & GVA & GVA \\
\hline $5 \mathrm{~km}$ & 773 & 1526 & 906 & 1409 & 765 & 1414 \\
\hline $15 \mathrm{~km}$ & 438 & 826 & 511 & 770 & 434 & 772 \\
\hline $50 \mathrm{~km}$ & 226 & 376 & 258 & 358 & 224 & 359 \\
\hline $100 \mathrm{~km}$ & - & - & 166 & 212 & 146 & 213 \\
\hline
\end{tabular}

Table 2. Resonance frequencies (in $\mathrm{Hz}$ ) for the combination cable - grid: lumped cable capacitance

\begin{tabular}{|l|c|c|c|c|c|c|}
\hline & \multicolumn{2}{|c|}{$132 \mathrm{kV}$} & \multicolumn{2}{c|}{$275 \mathrm{kV}$} & \multicolumn{2}{c|}{$400 \mathrm{kV}$} \\
\hline & $\begin{array}{c}1 \\
\text { GVA }\end{array}$ & $\begin{array}{c}4.1 \\
\text { GVA }\end{array}$ & $\begin{array}{c}6 \\
\text { GVA }\end{array}$ & $\begin{array}{c}15 \\
\text { GVA }\end{array}$ & $\begin{array}{c}9 \\
\text { GVA }\end{array}$ & $\begin{array}{c}32 \\
\text { GVA }\end{array}$ \\
\hline $5 \mathrm{~km}$ & 780 & 1580 & 917 & 1450 & 772 & 1460 \\
\hline $15 \mathrm{~km}$ & 450 & 912 & 529 & 837 & 446 & 840 \\
\hline $50 \mathrm{~km}$ & 247 & 500 & 290 & 459 & 244 & 461 \\
\hline $100 \mathrm{~km}$ & - & - & 205 & 324 & 173 & 326 \\
\hline
\end{tabular}

The two models give resonance frequencies that are somewhat similar. However, especially for longer cables, the differences are too big for the simple model to be of practical use to predict distortion levels. The simple model with lumped capacitances (equation (8) and Table 2) will however give an impression about the frequency range in which the resonance frequency can be expected. It may be used as a tool to decide about the need for more detailed studies. When the resonance frequency obtained from the simple model is less than $700 \mathrm{~Hz}$ (in a $50-\mathrm{Hz}$ system), resonances at harmonic 13 or lower can be expected and it is recommended to perform additional studies using detailed models. These additional studies should use the distributed parameter model and further 
details of the transmission grid. Recommendations about how to model the transmission grid for harmonic studies are given among others in $[3,4,5,6]$.

\section{Case study - cable plus grid}

The transfer function, input impedance and transfer impedance have been calculated for a $275-\mathrm{kV}$ cable. The parameters used have been the same as before, with the difference that the cable resistance has been calculated as a function of frequency:

$$
\mathbf{r}=\frac{0.082}{D_{m m}} \times \sqrt{f}
$$

With $D_{m m}=500 \mathrm{~mm}^{2}$, the cable cross section. Here it should be noted that other, more complicated expressions exist to model the increase of series resistance with frequency. See for example the overview given in [3].

The three functions defined in (5), (6) and (7) have been calculated for the base case as defined in Section 3, and for three other grid strengths; with the results shown in Figure 2. The resonance frequency increases with increasing grid strength as the inductive part of the source impedance becomes smaller. The peak values of input impedance on sending end (center plot) and the transfer impedance from sending to receiving end (bottom) are not much dependent on the grid strength. The transfer function from sending to receiving end does however show a large increase with increasing grid strength. Thus the same harmonic source (on sending end) and the same cable length will give a higher harmonic current at the receiving end for a strong grid than for a weak grid. The resulting harmonic voltage (determined by the transfer impedance) will be about the same.
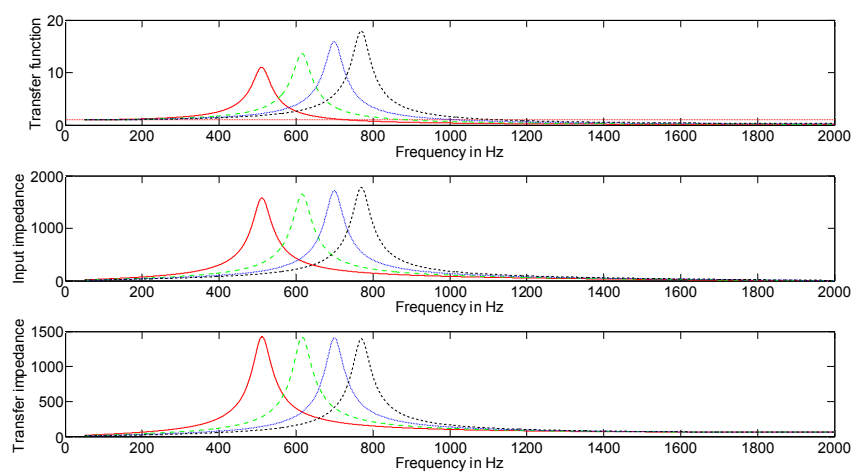

Figure 2. Transfer function (top); input impedance (center) and transfer impedance (bottom) for a $15 \mathrm{~km}$ cable connected to a $275 \mathrm{kV}$ grid with fault level $6000 \mathrm{MVA}$ (red solid), $9000 \mathrm{MVA}$ (green dashed), $12000 \mathrm{MVA}$ (blue dotted) and $15000 \mathrm{MVA}$ (black dash-dotted).

The impact of the cable length is shown in Figure 3. Increasing cable length shifts the resonance to lower frequencies as longer cables correspond to more capacitance. Longer cables also correspond to more resistance, hence the decrease in maximum transfer and impedance for increasing cable length.

With a cable length of $30 \mathrm{~km}$, a second resonance peak occurs around $1900 \mathrm{~Hz}$. This second resonance frequency is not predicted by the first-order model; instead it is a direct consequence of the distributed character of cables. Cables, as well as transmission lines, have an infinite number of resonance frequencies; but for most practical purposes only the first few resonances are of interest.
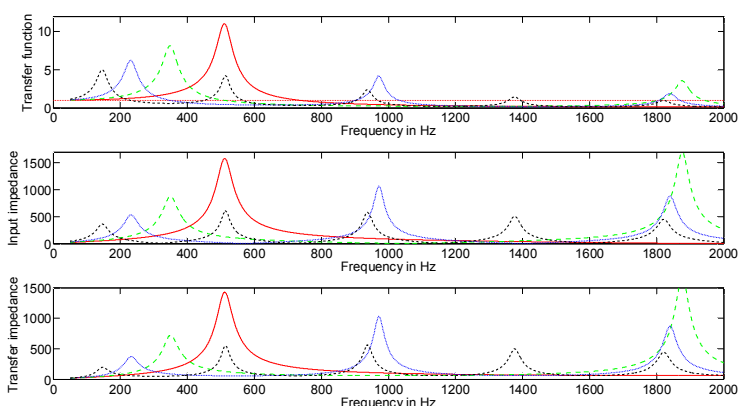

Figure 3. Transfer function (top) ; input impedance (center) and transfer impedance (bottom) for a cable connected to a $275 \mathrm{kV}$ grid with fault level 6000 MVA; $15 \mathrm{~km}$ (red solid), $30 \mathrm{~km}$ (green dashed), $60 \mathrm{~km}$ (blue dotted) and $120 \mathrm{~km}$ (black dashdotted).

The impact of the $\mathrm{X} / \mathrm{R}$ ratio is illustrated in Figure 4 .

Despite that the $\mathrm{X} / \mathrm{R}$ ratio varies only over a small range (from 0.8 to 1.4), the amplification around the resonance frequency varies a lot. The impact is however only noticeable around the resonance frequency. For the example shown here, the impact is big for harmonic 10 $(500 \mathrm{~Hz})$ but small for harmonics $9(450 \mathrm{~Hz})$ and 11 $(550 \mathrm{~Hz})$.
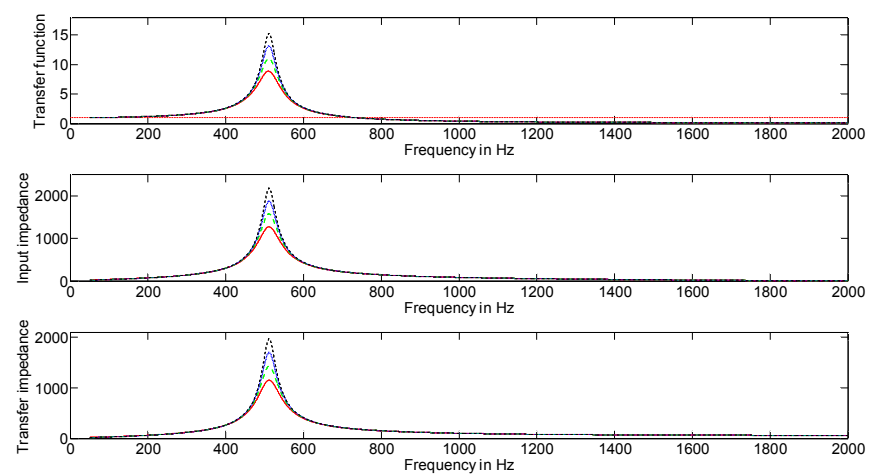

Figure 4. Transfer function (top) ; input impedance (center) and ransfer impedance (bottom) for a $15 \mathrm{~km}$ cable connected to a $275 \mathrm{kV}$ grid; source $\mathrm{X} / \mathrm{R}$ ratio equal to 0.8 (red solid), 1.0 'green dashed), 1.2 (blue dotted) and 1.4 (black dash-dotted).

[n this example the resonance frequency does not correspond with a major harmonic, but for a $30 \mathrm{~km}$ cable, the resonance frequency will be close to harmonic 7 (350 $\mathrm{Hz}$ ) and the amplification of the seventh harmonic will vary between 6.5 and 11.2 times for the given variation in $\mathrm{X} / \mathrm{R}$ ratio.

In practical studies, it is not the $\mathrm{X} / \mathrm{R}$ ratio that needs to be known. Here it was only used to illustrate the impact of different amounts of damping. What needs to be known if the increase of resistance with frequency for different power-system components.

The resistive part of the source is determined not only by the resistance of the grid components, but also by the 
resistive part of the load; where both are frequency dependent.

The increase of cable resistance with frequency was already mentioned at the beginning of this section. A similar increase occurs for overhead lines. The good news is that the skin effect, which is mainly responsible for this increase, can be mathematically modelled for symmetrical configurations like circular conductors. (Equation (9) is an approximation of more accurate and much more complicated expressions.) The proximity effect, which may play an important role for cable conductors, is already much harder to model.

The increase of resistance with frequency is very difficult to model for transformers and synchronous generators. Often an exponential increase of resistance with frequency is used, with the exponent somewhere between 0.5 and 1.5. Finally, when it comes to the modelling of the load, there is no consensus on how to model this as a function of frequency. Different models are recommended in the different publications. Some reference is made to measurements in [4], but these measurements were made many years ago.

\section{Case study - capacitor bank at lower voltage level}

As a second case, the transfer of harmonics to a lower voltage level has been studied. The cable in this case is connected at $400 \mathrm{kV}$, and the transfer to $132 \mathrm{kV}$ is studied. A capacitor bank is connected at $132 \mathrm{kV}$. The base case is shown in Figure 5.

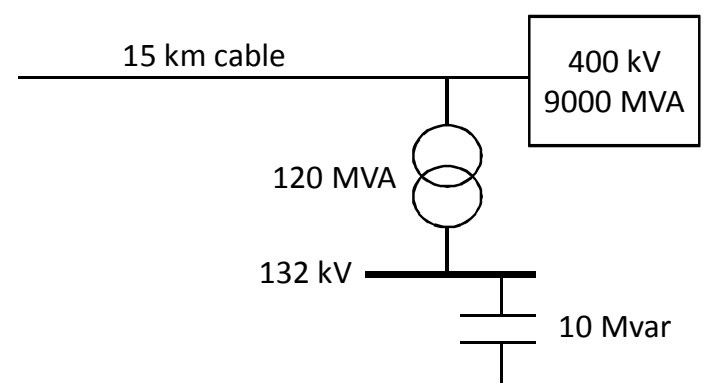

Figure 5. Base case configuration for studying transfer from 400 $\mathrm{kV}$ to $132 \mathrm{kV}$

The resulting transfer function (from sending end to receiving end of the cable) and the transfer impedance (from sending end of the cable to the terminals of the capacitor at $132 \mathrm{kV}$ ) are shown in Figure 6 for four different sizes of the capacitor bank. Compared to the case without capacitor bank, we see that two resonance frequencies occur at relatively low frequencies. For the base case (10 Mvar, black dashdot), the resonances occur around 300 and $600 \mathrm{~Hz}$. A third resonance occurs at $3700 \mathrm{~Hz}$ (not show here), which is independent of the size of the capacitor bank, in frequency and in magnitude.
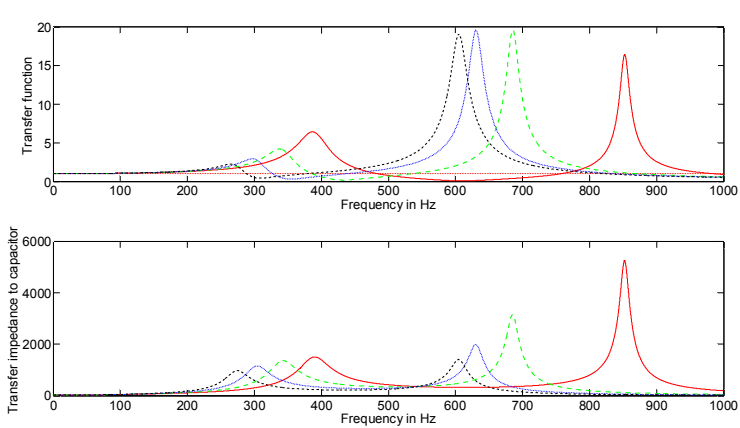

Figure 6. Transfer function from sending end to receiving end of the $400-\mathrm{kV}$ cable (top) and transfer impedance from sending end of the $400-\mathrm{kV}$ cable to the $132-\mathrm{kV}$ bus (bottom): capacitor bank 2.5 Mvar (red solid), 5 Mvar (green dashed), 7.5 Mvar (blue dotted) and 10 Mvar (black dashdot).

A decreasing size of the capacitor bank results in higher resonance frequencies and also in higher transfer to the receiving end, for the first resonance frequency. The amplitude of the second resonance frequency remains more constant.

In the transfer to the capacitor bank, both the first and the second resonance frequency increase in magnitude with reducing size of the capacitor bank.

The impact of the cable length is shown in Figure 7. Like for the case without capacitor bank, longer cables introduce more damping to the system, with a lower amplification at the resonance frequency as a result. Also here multiple resonances appear at higher frequencies, the one at $985 \mathrm{~Hz}$ for $60 \mathrm{~km}$ cable length is just visible in the figures. The one at $3700 \mathrm{~Hz}$ (mentioned earlier) shows a small change with cable length, but the other ones vary strongly with cable length.
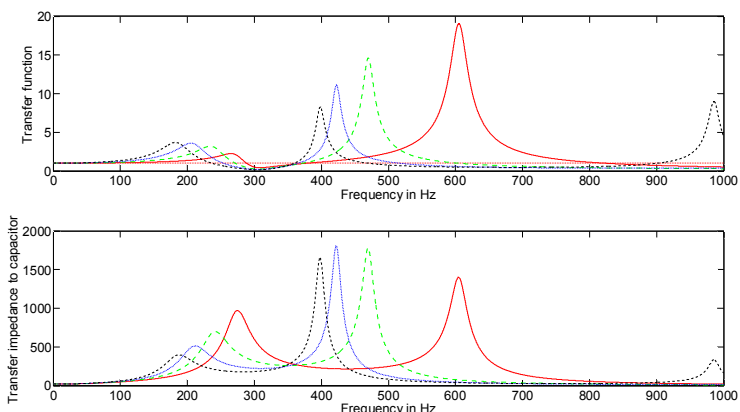

Figure 7. Transfer function from sending end to receiving end of the $400-\mathrm{kV}$ cable (top) and transfer impedance from sending end of the $400-\mathrm{kV}$ cable to the $132-\mathrm{kV}$ bus (bottom): cable length $15 \mathrm{~km}$ (red solid), $30 \mathrm{~km}$ (green dashed), $45 \mathrm{~km}$ (blue dotted) and $60 \mathrm{~km}$ (black dashdot).

\section{About harmonic studies}

Harmonic studies at transmission level are normally done when new distortion loads or equipment is connected to the grid. This can be large industrial installations, but also HVDC links.

Normally, no studies are done when changes are made in the transmission grid. With the installation of long ac cables at transmission voltages it is recommended to 
perform such studies whenever potential resonance frequencies get near harmonic 13 or lower.

A suggested first step is to obtain a rough estimate of the resonance frequency using expression (9). This calculation is very easy and the data needed is limited to the fault level at both terminals of the cable, the cable length and the cable capacitance per unit length. The latter can even be estimated from data books when no exact data is available.

When the resulting resonance frequency is less than $700 \mathrm{~Hz}$ in a $50 \mathrm{~Hz}$ system $(840 \mathrm{~Hz}$ in a $60 \mathrm{~Hz}$ system $)$ it is recommended to do a detailed harmonic study, with the same level of accuracy as would be done for the connection of a new customer or HVDC installation.

With resonance frequencies below harmonic 14, the emission coming from lower voltage levels can be amplified due to cable resonances. The main harmonics that pass from the lower voltage levels to the transmission system are orders 5, 7, 11 and 13. Every transformer to lower voltage levels becomes in that case a source of harmonics. For all such transformers in the neighbourhood of the cable, the input impedance, transfer functions and transfer impedances should be calculated. For transfer functions and transfer impedances this could involve many calculations. Fortunately, there are several power-system analysis packages that include a good harmonics tool. Once the system has been modelled it is relatively easy to obtain these impedances and transfer functions.

The same issues are relevant with such studies as with harmonic studies for the connection of new customers or installations: e.g. the extent of the network to be studied; unbalanced modelling of transmission lines; the increase of resistance with frequency; the modelling of the load. The increase of resistance with frequency and the load modelling are the weak points in most studies. This is where future work on research side is very much needed.

To study the possible amplification of harmonics coming from lower voltage levels, it is probably sufficient to model the positive and negative-sequence networks. Some research studies should be done to find out if there are cases where the coupling between sequence networks due to unbalances in the network could require the modelling of the zero-sequence network as well.

Another source of harmonics at transmission level is the magnetizing branch of power transformers. This gives all odd harmonics, including the triplen ones (3, 9, 15 etc). The levels of harmonics 5, 7, 11 and 13 due to this are lower than the ones coming through the transformer from lower voltage levels. However for harmonics 3 and 9, the magnetizing branch is the main source. Especially when the transmission system is operated at higher voltage levels than originally intended, harmonics 3 and 9 from the magnetising branch cannot be neglected. To study possible amplification of this, the zero-sequence network should be modelled, including earthing and earth-return. Also are typically the values for source inductance and cable capacitance different, so that the rough estimation of the resonance frequency (the above-mentioned first step) should be repeated as well.

There is lack of experience on possible non-linear phenomena when resonances appear around the third harmonic and amplification of harmonics from the transformer magnetising branch occurs.

\section{Conclusions}

It is important to consider the impact of transmission cables on the harmonic levels in the grid. When long cables are connected a harmonic study is recommended.

A simple criterion is proposed here to decide about the need for a detailed study. Such a study should have at least the same accuracy and detail as the harmonic studies done with the connection of new industrial customers or HVDC links.

Further research is needed to obtain appropriate models for the increase in resistance with frequency and for the load as seen from the transmission system. Research is also required on the need to include the zero-sequence network when studying the possible amplification of harmonics coming from lower voltage levels.

Another important research subject is the study of possible non-linear phenomena when resonances occur at transmission level close to harmonic 3 .

\section{REFERENCES}

[1] W. Wiechowski and P.B. Eriksen. Selected studies on offshore wind farm cable connections challenges and experience of the Danish TSO. IEEE Power and Energy Society General Meeting, July 2008.

[2] Math Bollen and Fainan Hassan, Integration of distributed generation in the power system, WileyIEEE Press, New York, 2011.

[3] J. Arrillaga and N.R. Watson. Power system harmonics, second edition. Wiley, Chichester, UK, 2003.

[4] CIGRE WG 36-05, Harmonics, characteristic parameters, method of study, estimates of existing values in the network, Electra No. 77, pp. 35-56, 1981.

[5] Task force on Harmonics Modeling and Simulation, "The modeling and simulation of the propagation of harmonics in electric power networks Part I: Concepts, models and simulation techniques," IEEE Transactions on Power Delivery, Vol.11, No.1, January 1996, pp. 452-465.

[6] Task force on Harmonics Modeling and Simulation, "The modeling and simulation of the propagation of harmonics in electric power networks Part II: Sample systems and Examples," IEEE Transactions on Power Delivery, Vol.11, No.1, January 1996, pp. 466-474 